\title{
Antiviral Activity of Fridericia formosa (Bureau) L. G. Lohmann (Bignoniaceae) Extracts and Constituents
}

\author{
Geraldo Célio Brandão, ${ }^{1}$ Erna G. Kroon, ${ }^{2}$ José D. Souza Filho, ${ }^{3}$ and Alaíde Braga Oliveira ${ }^{4}$ \\ ${ }^{1}$ Departamento de Farmácia, Escola de Farmácia, Universidade Federal de Ouro Preto, Campus Morro do Cruzeiro, \\ 35.400-000 Ouro Preto, MG, Brazil \\ ${ }^{2}$ Departamento de Microbiologia, ICB, Universidade Federal de Minas Gerais, Av. Antônio Carlos 6627, \\ 31.270-901 Belo Horizonte, MG, Brazil \\ ${ }^{3}$ Departamento de Química, ICEX, Universidade Federal de Minas Gerais, Av. Antônio Carlos 6627, \\ 31.270-901 Belo Horizonte, MG, Brazil \\ ${ }^{4}$ Departamento de Produtos Farmacêuticos, Faculdade de Farmácia, Universidade Federal de Minas Gerais, \\ Av. Antônio Carlos 6627, 31.270-901 Belo Horizonte, MG, Brazil \\ Correspondence should be addressed to Alaíde Braga Oliveira; alaidebraga@terra.com.br
}

Received 16 August 2016; Revised 23 November 2016; Accepted 15 February 2017; Published 29 May 2017

Academic Editor: Carlos E. P. Corbett

Copyright (C) 2017 Geraldo Célio Brandão et al. This is an open access article distributed under the Creative Commons Attribution License, which permits unrestricted use, distribution, and reproduction in any medium, provided the original work is properly cited.

\begin{abstract}
A phytochemical study of Fridericia formosa (Bignoniaceae) ethanol extracts of leaves, stems, and fruits was guided by in vitro assays against vaccinia virus Western Reserve (VACV-WR), human herpes virus 1 (HSV-1), murine encephalomyocarditis virus (EMCV), and dengue virus type 2 (DENV-2) by the MTT method. All the ethanol extracts were active against DENV-2, HSV-1, and VACV-WR with best results for the fruits extract against DENV-2 (SI > 38.2). For VACV-WR and HSV-1, EC50 values $>200 \mu \mathrm{g}$ $\mathrm{mL}^{-1}$ were determined, while no inhibition of the cytopathic effect was observed with EMCV. Five compounds were isolated and identified as the C-glucosylxanthones mangiferin (1), $2^{\prime}$-O-trans-caffeoylmangiferin (2), $2^{\prime}$-O-trans-coumaroylmangiferin (3), $2^{\prime}$ $O$-trans-cinnamoylmangiferin (5), and the flavonoid chrysin (4). The most active compound was $2^{\prime}$-O-trans-coumaroylmangiferin (3) with SI > 121.9 against DENV-2 and 108.7 for HSV-1. These results indicate that mangiferin cinnamoyl esters might be potential antiviral drugs.
\end{abstract}

\section{Introduction}

Viral infections represent a current problem accounting for severe damage to human health and economic losses in livestock [1]. Some viral diseases such as dengue or dengue fever (DF), herpes, smallpox, and encephalomyocarditis have a high impact in public health in the tropical and subtropical regions of the world [1].

Dengue virus belonging to the Flaviviridae family, Flavivirus genus, is responsible for Dengue fever (DF) and is considered the most common arboviral disease of humans. It is estimated that 390 million cases occur every year around the world and it is endemic in more than 100 countries, including the Americas, Southeast Asia, and Western Pacific, regions most seriously affected $[1,2]$. No effective drug as well as no vaccine is available for human use. The need for a safe and efficient approach either for treatment or prevention of DF has been considered a global priority $[1,3]$.

HSV belongs to the family Herpesviridae and the subfamily Alphaherpesvirinae and is characterized by neurovirulence, latency, and reactivation. The prevalence of HSV infection has increased in recent years, making it a highly relevant public health issue. Early detection and treatment are of paramount importance for disease control [4].

Encephalomyocarditis virus (EMCV) family Picornaviridae, genus Cardiovirus, is a group of closely related virus species with a wide host range. Infections with EMCV are associated with sporadic cases and outbreaks of myocarditis 
and encephalitis in domestic pigs, in nonhuman primates and other mammalian species. There are few reports of cases of human infection by EMCV [5].

Vaccinia (VACV) is a virus of the genus Orthopoxvirus of the family Poxviridae that, in humans, causes nonlethal, pustular, and localized disease. The vaccinia virus does not have natural hosts, but cases of bovine and human infection by vaccinia virus are reported in Brazil and India, causing economic losses and affecting health services $[6,7]$.

As part of a bioprospecting project, whose main goal is to discover potential antiviral natural products of plants from Brazilian Cerrado and Atlantic Forest biomes, we have screened several species of plants collected in the state of Minas Gerais [1, 8-12]. Among these, Fridericia formosa (Bureau) Sandwith was chosen for bioguided phytochemical investigation due to the good antiviral activity presented by the ethanol extracts of leaves, stems, and fruits.

\section{Materials and Methods}

2.1. Collection, Taxonomical Determination, and Processing of Plant Materials. F. formosa was collected in the municipality of Belo Horizonte, Minas Gerais, Brazil. The plant was taxonomically identified by Dr. J. A. Lombardi, Departamento de Botânica, Instituto de Biociências, UNESP, Rio Claro, Brazil. A voucher specimen was deposited at the BHCB/UFMG, Belo Horizonte, Minas Gerais, Brazil, under the number 23885.

2.2. Preparation of Extracts. After drying in air circulating oven at $40^{\circ} \mathrm{C}$ for $72 \mathrm{~h}$, the plant material-136.3 $\mathrm{g}$ of leaves, $461.5 \mathrm{~g}$ of stems, and $13.8 \mathrm{~g}$ fruits-were ground and extracted by percolation with $96 \% \mathrm{EtOH}$ at room temperature. The solvent was removed in a rotary evaporator under reduced pressure at $50^{\circ} \mathrm{C}$, leaving dark residues-EEFFL, $34.2 \mathrm{~g}$, EEFFS, $60.0 \mathrm{~g}$, and EEFFF, $2.5 \mathrm{~g}$-for leaves, stems, and fruits, respectively, which were kept in a vacuum desiccator until constant weight.

2.3. HPLC Analyses. In the HPLC analyses, an exploratory gradient elution was used $[1,9,10]$. Fingerprints were registered by RP-HPLC-DAD on a Waters 2695 apparatus equipped with a UV-DAD detector (Waters 2996). A LiChrospher 100 RP-18 column $(5 \mu \mathrm{m}, 250 \times 4 \mathrm{~mm}$ i.d.; Merck, Darmstadt, Germany) was employed at $40^{\circ} \mathrm{C}$, flow rate of $1.0 \mathrm{~mL} / \mathrm{min}$, and detection at wavelengths of 220,280 , and $350 \mathrm{~nm}$. To an aliquot $10.0 \mathrm{mg}$ of dried extract/fractions and $1.0 \mathrm{mg}$ of each of the isolated compounds HPLC grade methanol was added and the mixture was dissolved by sonication in an ultrasound bath for $15 \mathrm{~min}$, followed by centrifugation at $10,000 \mathrm{rpm}$ for $10 \mathrm{~min}$. The supernatant was filtered through a Millipore membrane $(0.2 \mu \mathrm{m})$ and injected $(10.0 \mu \mathrm{L})$ onto the equipment. Elution was carried out with a linear gradient of water (a) and acetonitrile (b) (from 5\% to $95 \%$ of B in $60 \mathrm{~min})$.

2.4. Isolation of Chemical Components from Leaves Extract. To a portion of EEFFL ( $10.0 \mathrm{~g}), \mathrm{MeOH}$ was added, and an insoluble precipitate was separated by filtration through sintered glass funnel and washed thoroughly with $\mathrm{MeOH}$ yielding $2.8 \mathrm{~g}$. The precipitate was recrystallized out from methanol/water $(1: 1)$ giving $1.9 \mathrm{~g}$ of compound 1 . The filtrate was dried in a rotary evaporator under reduced pressure at $50^{\circ} \mathrm{C}$, leaving a dark residue (FFLMW, $7.0 \mathrm{~g}$ ). AFLMW was subjected to fractionation over a silica gel column with n-hexane/ $\mathrm{CH}_{2} \mathrm{Cl}_{2}(1: 1), \mathrm{CH}_{2} \mathrm{Cl}_{2}, \mathrm{CH}_{2} \mathrm{Cl}_{2} / \mathrm{EtOAc}$ (1:1), EtOAc/MeOH $(2: 1)$, EtOAc/MeOH (1:2), $\mathrm{MeOH}$, $\mathrm{MeOH} / \mathrm{H}_{2} \mathrm{O}(2: 1)$, and $\mathrm{MeOH} / \mathrm{H}_{2} \mathrm{O}(1: 2)$ as eluents. A portion of EtOAc/MeOH $(2: 1)$ fraction 2 (1.0 g) was filtered over a Sephadex LH 20 column with $\mathrm{MeOH}$ as eluent, giving 12 fractions. Fractions 11 and 12 were combined leading to a yellow solid $(554.0 \mathrm{mg}$ ) which was further purified by HPLC employing Shim-pack PRC-ODS column $(20 \mathrm{~mm}$ i.d. $\times 25 \mathrm{~cm}$, flow rate $5.0 \mathrm{ml} / \mathrm{min}$ ) and a gradient elution with $\mathrm{MeOH} / \mathrm{H}_{2} \mathrm{O} 45$ to $95 \%$ for 50 minutes. Compounds 2 $(22.3 \mathrm{mg})$ and $\mathbf{3}(56.1 \mathrm{mg})$ were isolated.

2.5. Isolation of Chemical Components from Stem Extract. To a portion of EEFFS $(10.0 \mathrm{~g})$, cold $\mathrm{MeOH}$ was added giving a precipitate which was separated by filtration on sintered glass funnel and washed thoroughly with $\mathrm{MeOH}$ yielding $2.43 \mathrm{~g}$. The precipitate was recrystallized out twice from $\mathrm{MeOH}$ to give $405.0 \mathrm{mg}$ of compound $\mathbf{1}$. The mother liquor from the second recrystallization was dried in a rotary evaporator under reduced pressure at $50^{\circ} \mathrm{C}$, leaving a yellow residue (FFSMW2, $1.2 \mathrm{~g}$ ) that was further subjected to fractionation by HPLC employing Shim-pack PRC-ODS column $(20 \mathrm{~mm}$ i.d. $\times 25 \mathrm{~cm}$, flow rate $5.0 \mathrm{ml} / \mathrm{min}$ ) and a gradient elution with $\mathrm{MeOH} / \mathrm{H}_{2} \mathrm{O} 45$ to $95 \%$ for 50 minutes affording compound 4 (511.0 mg).

2.6. Isolation of Chemical Components from Fruit Extract. To a portion of EEFFF $(1.0 \mathrm{~g}), \mathrm{MeOH}$ was added and an insoluble precipitate was separated by filtration on sintered glass funnel and washed thoroughly with methanol. The precipitate was subjected to recrystallization to give compound 1 ( $80.7 \mathrm{mg}$.) The filtrate was dried in a rotary evaporator under reduced pressure at $50^{\circ} \mathrm{C}$, leaving a yellow residue (FFFMW, $809.7 \mathrm{mg}$ ) that was further purified by HPLC employing Shim-pack PRC-ODS column $(20 \mathrm{~mm}$ i.d. $\times 25 \mathrm{~cm}$, flow rate $5.0 \mathrm{ml} / \mathrm{min}$ ) and a gradient elution with $\mathrm{MeOH} / \mathrm{H}_{2} \mathrm{O} 45$ to $95 \%$ for 50 minutes leading to the isolation of compounds 1 (96.3 mg), 2 (13.4 mg), 3 (82.9 mg), and 5 (84.6 mg).

2.7. Cell Culture and Virus. Vero cells (ATCC CCL-81) and LLCMK ${ }_{2}$ cells were cultured in Dulbecco's modified Eagle's medium (DMEM, Cultilab, Campinas, SP, Brazil) at $37^{\circ} \mathrm{C}$, in $5 \% \mathrm{CO}_{2}$ atmosphere, supplemented with $5 \%$ fetal bovine serum, $50 \mu \mathrm{g} / \mathrm{mL}$ gentamicin, $100 \mathrm{U} / \mathrm{mL}$ penicillin, and $5 \mu \mathrm{g} / \mathrm{mL}$ amphotericin B [1].

HSV-1 was obtained from the collection of Laboratório de Virus, UFMG, Belo Horizonte, Brazil. DENV-2, EMCV, and VACV-WR were kindly donated by Dr. L. Figueiredo (USP, Ribeirão Preto, Brazil), Dr. I. Kerr (London Research Institute, London, UK), and Dr. C. Jungwirth (University of 
Würzburg, Würzburg, Germany), respectively. The viruses were titrated by TCID $_{50}$ in Vero cells [13] and the titers were $2.5 \times 10^{6} ; 1.0 \times 10^{6} ; 1.0 \times 10^{6}$; and $1.0 \times 10^{4} \mathrm{TCID}_{50} / \mathrm{mL}$, respectively, for HSV-1, EMCV, VACV-WR, and DENV-2.

2.8. Cytotoxicity Assay. Vero and $\mathrm{LLCMK}_{2}$ cells were exposed to different concentrations of extracts/fractions/compounds for 48 and $72 \mathrm{~h}$ [1]. After incubation, cell viability was assessed by the 3-(4,5-dimethylthiazol-2-yl)-2,5-diphenyltetrazolium bromide (MTT, Merck) assay at a concentration of $2 \mathrm{mg} / \mathrm{mL}$ in PBS $[1,14]$. Each sample was assayed in four replicates for concentrations ranging from 500 to $0.125 \mu \mathrm{g} / \mathrm{mL}$. The cytotoxicity of each sample was expressed as $\mathrm{CC}_{50}$, that is, the concentration of sample that inhibited cell growth by $50 \%[1]$.

2.9. Antiviral Assays. The antiviral activity $\left(\mathrm{EC}_{50}\right)$ of extracts \fractions \compounds was evaluated by the MTT assay [15]. Acyclovir (Calbiochem, Merck Brasil, São Paulo, SP, Brazil) and $\alpha$-2a interferon (Bergamo Brasil, São Paulo, SP, Brazil) were used as positive controls [1]. The cell monolayer was infected with viral suspensions with titers of $2.5 \times 10^{6}$; $1.0 \times 10^{6} ; 1.0 \times 10^{6}$; and $1.0 \times 10^{4} \mathrm{TCID}_{50} / \mathrm{mL}$, respectively, for HSV-1, EMCV, VACV-WR, and DENV-2 [1]. Dilutions of the extracts, fractions, and compounds in noncytotoxic concentrations were added to the wells after viral infection. The plates were incubated at $37^{\circ} \mathrm{C}$ in humidified $5 \% \mathrm{CO}_{2}$ atmosphere for a period of 48 and/or $72 \mathrm{~h}$ [1]. Experiments were carried out with eight different concentrations within the inhibitory range of the samples. The 50\% inhibitor concentrations of the viral effect $\left(\mathrm{EC}_{50}\right)$ for each of the extracts, fractions, and constituents were calculated from concentration-effect-curves after no linear regression analysis [1]. The selectivity index (SI) is defined as $\mathrm{CC}_{50}$ over $\mathrm{EC}_{50}$. Statistical calculations were carried out with the GraphPad prism 5.0 software package (Statistica). Results are expressed as the mean \pm SEM of 4 independent experiments. Student's $t$-test was used for statistical analyses; $P$ values $>0.05$ were considered to be significant.

2.10. Structural Determination. The compounds isolated were identified on the basis of spectral analyses and comparison with literature data. ${ }^{1} \mathrm{D}$ and ${ }^{2} \mathrm{D}{ }^{1} \mathrm{H}$ and ${ }^{13} \mathrm{C}$-NMR spectra such as COSY, HSQC, and HMBC were obtained on a Bruker Avance DRX400 instrument in DMSO-d6 with TMS as internal standard. Chemical shifts are given as $\delta$ (ppm). LC-MS data were obtained by electrospray ionization mass spectrometry (ESI-MS) in an Esquire 3000 Plus Bruker Daltonics equipment, capillary: $4000 \mathrm{~V}$, nebulizer: $27 \mathrm{psi}$, dry gas: $7.0 \mathrm{~L} / \mathrm{min}$, dry temp: $320^{\circ} \mathrm{C}$, and mass flux $100 \mathrm{uL} / \mathrm{min}$, in the Central Analítica, Instituto de Química, Universidade de São Paulo, São Paulo, SP, Brazil [1].

\subsection{Spectroscopic Data for Isolated Compounds}

Mangiferin (1; 2- $\beta$-D-Glucopyranosyl-1,3,6,7-tetrahydroxy9H-xanthen-9-one). orange powder $(\mathrm{MeOH}) ;$ m.p. decomposes at $265.0-275.0$; Lit. $271-274^{\circ} \mathrm{C}$ [16]; UV (MeOH) $\lambda_{\max }$
239, 258, 316, $365 \mathrm{~nm}$; IR $v_{\text {max }} 3363,3184,2935,2891,1648$, $1619,1592,1565,1520,1490,1462,1406,1351,1294,1251,1191$, 1093, 1074, 1032, 878, 822, $735 \mathrm{~cm}^{-1}$; ${ }^{1} \mathrm{H}$ NMR (DMSO-d6, $400 \mathrm{MHz}): \delta 13.77$ (s, 1H, 1-OH), 10.55 (s, 2H, 6,7-OH), 7.38 (s, 1H, H-8), 6.86 (s, 1H, H-5), 6.37 (s, 1H, H-4), 4.87 (s, 2H, $\left.3^{\prime}, 4^{\prime}-\mathrm{OH}\right), 4.61\left(\mathrm{~d}, 10.0 \mathrm{~Hz}, 1 \mathrm{H}, \mathrm{H}-1^{\prime}\right), 4.49\left(\mathrm{~s}, 1 \mathrm{H}, 6^{\prime}-\mathrm{OH}\right), 4.05$ $\left(\mathrm{t}, 8.4 \mathrm{~Hz}, 1 \mathrm{H}, \mathrm{H}-2^{\prime}\right), 3.70\left(\mathrm{~d}, 11.2 \mathrm{~Hz}, 1 \mathrm{H}, \mathrm{H}-6^{\prime}{ }_{\mathrm{B}}\right), 3.41(\mathrm{~m}, 1 \mathrm{H}$, H-6 $\left.{ }_{\mathrm{A}}\right), 3.18\left(\mathrm{~m}, 1 \mathrm{H}, \mathrm{H}-3^{\prime}\right), 3.18\left(\mathrm{~m}, 1 \mathrm{H}, \mathrm{H}-4^{\prime}\right), 3.18(\mathrm{~m}, 1 \mathrm{H}, \mathrm{H}-$ $\left.5^{\prime}\right) .{ }^{13} \mathrm{C}$ NMR (DMSO-d6, $\left.100 \mathrm{MHz}\right): \delta 179.1$ (C, C-9), 163.8 (C, C-3), 161.8 (C, C-1), 156.2 (C, C-4a), 154.0 (C, C-6), 150.8 (C, C-10a), 143.7 (C, C-7), 111.7 (C, C-8a), 108.1 (C, C-8), 107.5 (C, C-2), 102.6 (C, C-5), 93.3 (C, C-4), 81.5 (C, C-5'), 79.3 (C, C-3'), $73.1\left(\mathrm{C}, \mathrm{C}-1^{\prime}\right), 70.6\left(\mathrm{C}, \mathrm{C}-4^{\prime}\right), 70.3\left(\mathrm{C}, \mathrm{C}-2^{\prime}\right), 61.5(\mathrm{C}, \mathrm{C}-$ $\left.6^{\prime}\right)$; HRESI-MS $m / z 423.1024[\mathrm{M}-\mathrm{H}]^{-}\left(\right.$calcd for $\mathrm{C}_{19} \mathrm{H}_{19} \mathrm{O}_{11}$, 423.0927).

$2^{\prime}$-O-trans-caffeoylmangiferin (2; 2-(2'-O-trans-caffeoyl)-C$\beta$-D-glucopyranosyl-1,3,6,7-tetrahydroxyxanthone). orange powder $(\mathrm{MeOH}) ; \mathrm{m} . \mathrm{p}$. decomposes at $269.0-278.0^{\circ} \mathrm{C}$; UV $(\mathrm{MeOH}) \lambda_{\max } 232,258,315,365 \mathrm{~nm}$; IR $\nu_{\max } 3217,1690,1604$, 1514, 1471, 1259, 1150, 1071, $812 \mathrm{~cm}^{-1}$; ${ }^{1} \mathrm{H}$ NMR (DMSO-d6, $400 \mathrm{MHz}): \delta 13.83$ (s, 1H, 1-OH), 7.33 (s, $1 \mathrm{H}, \mathrm{H}-8), 7.23$ (d, $\left.16.0 \mathrm{~Hz}, 1 \mathrm{H}, \mathrm{H}-7^{\prime \prime}\right), 6.92\left(\mathrm{~m}, 1 \mathrm{H}, \mathrm{H}-2^{\prime \prime}\right), 6.87(\mathrm{~d}, 8.0 \mathrm{~Hz}, 1 \mathrm{H}$, H-6" $\left.{ }^{\prime \prime}\right), 6.80(\mathrm{~s}, 1 \mathrm{H}, \mathrm{H}-5), 6.71\left(\mathrm{~d}, 8.0 \mathrm{~Hz}, 1 \mathrm{H}, \mathrm{H}-5^{\prime \prime}\right), 6.30(\mathrm{~s}$, $1 \mathrm{H}, \mathrm{H}-4), 6.01\left(\mathrm{~d}, 16.0 \mathrm{~Hz}, 1 \mathrm{H}, \mathrm{H}-8^{\prime \prime}\right), 5.65\left(\mathrm{~m}, 1 \mathrm{H}, \mathrm{H}-2^{\prime}\right), 4.95$ $\left(\mathrm{d}, 12.0 \mathrm{~Hz}, 1 \mathrm{H}, \mathrm{H}-\mathrm{I}^{\prime}\right), 3.76\left(\mathrm{~m}, 1 \mathrm{H}, \mathrm{H}-6^{\prime}{ }_{\mathrm{B}}\right), 3.74(\mathrm{~m}, 1 \mathrm{H}, \mathrm{H}-$ $\left.6^{\prime}{ }_{\mathrm{A}}\right), 3.49\left(\mathrm{~m}, 1 \mathrm{H}, \mathrm{H}-3^{\prime}\right), 3.45\left(\mathrm{~m}, 1 \mathrm{H}, \mathrm{H}-4^{\prime}\right), 3.27(\mathrm{~m}, 1 \mathrm{H}, \mathrm{H}-$ $5^{\prime}$ ). ${ }^{13} \mathrm{C}$ NMR (DMSO-d6, $\left.100 \mathrm{MHz}\right): \delta 179.0$ (C, C-9), 165.2 (C, C-9" $\left.{ }^{\prime \prime}\right), 164.4$ (C, C-3), 161.5 (C, C-1), 156.3 (C, C-4a), 154.5 (C, C-6), 150.8 (C, C-10a), 148.2 (C, C-4" $\left.4^{\prime \prime}\right), 145.9\left(\mathrm{C}, \mathrm{C}-3^{\prime \prime}\right)$, $144.9\left(\mathrm{C}, \mathrm{C}-7^{\prime \prime}\right), 143.9$ (C, C-7), $125.4\left(\mathrm{C}, \mathrm{C}-1^{\prime \prime}\right), 121.6$ (C, C6"), 115.7 (C, C-5"), 115.0 (C, C-2"), 114.2 (C, C-8 $\left.{ }^{\prime \prime}\right), 111.4$ (C, C-8a), 107.8 (C, C-8), 105.7 (C, C-2), 102.5 (C, C-5), 94.2 (C, C4), $82.3\left(\mathrm{C}, \mathrm{C}-5^{\prime}\right), 76.9\left(\mathrm{C}, \mathrm{C}-3^{\prime}\right), 72.4\left(\mathrm{C}, \mathrm{C}-2^{\prime}\right), 71.1\left(\mathrm{C}, \mathrm{C}-1^{\prime}\right)$, $71.1\left(\mathrm{C}, \mathrm{C}-4^{\prime}\right), 61.9\left(\mathrm{C}, \mathrm{C}-6^{\prime}\right)$; HRESI-MS $m / z 583.1083$ [M $\mathrm{H}]^{-}$(calcd for $\mathrm{C}_{28} \mathrm{H}_{23} \mathrm{O}_{14}$, 583.1088).

$2^{\prime}$-O-trans-coumaroylmangiferin (3; 2-(2'-O-trans-coumaroyl)-C- $\beta$-D-glucopyranosyl-1,3,6,7-tetrahydroxyxanthone). orange powder $(\mathrm{MeOH}) ;$ m.p. decomposes at 271.0-285.0; UV (MeOH) $\lambda_{\max } 233,258,315,364 \mathrm{~nm}$; IR $\nu_{\max } 3255,1694$, 1614, 1472, 1417, 1365, 1284, 1230, 1150, 1079, 1030, 996, 815, 765, 706, $681 \mathrm{~cm}^{-1} ;{ }^{1} \mathrm{H}$ NMR (DMSO-d6, $400 \mathrm{MHz}$ ): $\delta 13.86$ (s, 1H, 1-OH), 7.39 (s, 1H, H-8), 7.37 (d, $\left.16.0 \mathrm{~Hz}, 1 \mathrm{H}, \mathrm{H}-7^{\prime \prime}\right)$, $7.35\left(\mathrm{~d}, 8.0 \mathrm{~Hz}, 1 \mathrm{H}, \mathrm{H}-2^{\prime \prime}\right), 7.35\left(\mathrm{~d}, 8.0 \mathrm{~Hz}, 1 \mathrm{H}, \mathrm{H}-6^{\prime \prime}\right), 6.81$ $(\mathrm{s}, 1 \mathrm{H}, \mathrm{H}-5), 6.76\left(\mathrm{~d}, 8.0 \mathrm{~Hz}, 1 \mathrm{H}, \mathrm{H}-3^{\prime \prime}\right), 6.76(\mathrm{~d}, 8.0 \mathrm{~Hz}, 1 \mathrm{H}$, H-5 $\left.5^{\prime \prime}\right), 6.32(\mathrm{~s}, 1 \mathrm{H}, \mathrm{H}-4), 6.10\left(\mathrm{~d}, 16.0 \mathrm{~Hz}, 1 \mathrm{H}, \mathrm{H}-8^{\prime \prime}\right), 5.56$ $\left(\mathrm{m}, 1 \mathrm{H}, \mathrm{H}-2^{\prime}\right), 5.01\left(\mathrm{~d}, 8.0 \mathrm{~Hz}, 1 \mathrm{H}, \mathrm{H}-\mathrm{l}^{\prime}\right), 3.78(\mathrm{~d}, 8.0 \mathrm{~Hz}, 1 \mathrm{H}$, $\left.\mathrm{H}-6_{\mathrm{B}}^{\prime}\right), 3.66\left(\mathrm{~m}, 1 \mathrm{H}, \mathrm{H}-6^{\prime}{ }_{\mathrm{A}}\right), 3.64\left(\mathrm{~m}, 1 \mathrm{H}, \mathrm{H}-3^{\prime}\right), 3.47(\mathrm{t}$, $\left.10.0 \mathrm{~Hz}, 1 \mathrm{H}, \mathrm{H}-4^{\prime}\right), 3.42\left(\mathrm{~m}, 1 \mathrm{H}, \mathrm{H}-5^{\prime}\right) .{ }^{13} \mathrm{C}$ NMR (DMSO-d6, $100 \mathrm{MHz}): \delta, 165.8$ (C, C-9" $), 164.0$ (C, C-3), 160.0 (C, C-1), $160.0\left(\mathrm{C}, \mathrm{C}-4^{\prime \prime}\right), 158.7$ (C, C-4a), 154.0 (C, C-6), 151.3 (C, C-10a), 144.5 (C, C-7") 143.9 (C, C-7), 130.1 (C, C-2" ), 130.1 (C, C-6"), 125.4 (C, C-1" ), 115.9 (C, C-3"), 115.9 (C, C-5" ), 114.4 (C, C-8" ), 112.2 (C, C-8a), 108.3 (C, C-8), 105.5 (C, C-2), 102.8 (C, C-5), 94.0 (C, C-4), 81.4 (C, C-5'), $76.8\left(\mathrm{C}, \mathrm{C}-3^{\prime}\right)$, $72.5\left(\mathrm{C}, \mathrm{C}-2^{\prime}\right), 71.4\left(\mathrm{C}, \mathrm{C}-1^{\prime}\right), 70.7\left(\mathrm{C}, \mathrm{C}-4^{\prime}\right), 61.4\left(\mathrm{C}, \mathrm{C}-6^{\prime}\right)$; HRESI-MS $m / z 567.1144[\mathrm{M}-\mathrm{H}]^{-}\left(\right.$calcd for $\mathrm{C}_{28} \mathrm{H}_{23} \mathrm{O}_{13}$, 567.1139). 
Chrysin (4; 5,7-Dihydroxy-2-phenyl-4H-1-benzopyran-4-one). orange powder $(\mathrm{MeOH})$; m.p. $285.6-287.9^{\circ} \mathrm{C}$; Lit. $289-291^{\circ} \mathrm{C}$ [17]; UV (MeOH) $\lambda_{\max } 267,313$ (sh) nm; IR $\nu_{\max } 3283,2920$, 1690, 1610, 1512, 1444, 1341, 1230, 1171, 1066, 1037, 1012, 893, $823 \mathrm{~cm}^{-1}$; ${ }^{1} \mathrm{H}$ NMR (DMSO-d6, $400 \mathrm{MHz}$ ): $\delta 12.0$ (s, 1H, 5$\mathrm{OH}) ; 8.06\left(\mathrm{dd}, 6.4\right.$ and $1.6 \mathrm{~Hz}, 2 \mathrm{H}, \mathrm{H}-2^{\prime}$ and $\left.\mathrm{H}-6^{\prime}\right), 7.55-7.64$ (m, 3H, H-3', $\mathrm{H}-4^{\prime}$ and $\left.\mathrm{H}-5^{\prime}\right) ; 6.95$ (s, 1H, H-3); 6.86 (s, $1 \mathrm{H}$, 7-OH); 6.53 (d, $2 \mathrm{~Hz}, 1 \mathrm{H}, \mathrm{H}-8) ; 6.23$ (d, $2 \mathrm{~Hz}, 1 \mathrm{H}, \mathrm{H}-6) .{ }^{13} \mathrm{C}$ NMR (DMSO-d6, $100 \mathrm{MHz}$ ): $\delta 181.9$ (C, C-4), 164.4 (C, C-7), 163.2 (C, C-2), 161.5 (C, C-5), 157.5 (C, C-9), 132.0 (C, C-4'), $130.7\left(\mathrm{C}, \mathrm{C}-1^{\prime}\right), 129.4\left(\mathrm{C}, \mathrm{C}-5^{\prime}\right), 129.1\left(\mathrm{C}, \mathrm{C}-3^{\prime}\right), 126.4\left(\mathrm{C}, \mathrm{C}-2^{\prime}\right)$, 126.4 (C, C-6' ${ }^{\prime}$ ), 105.2 (C, C-3), 104.0 (C, C-10), 99.0 (C, C-6), 94.1 (C, C-8); HRESI-MS $\mathrm{m} / z$ 255.0671 [M + H $]^{+}($calcd for $\left.\mathrm{C}_{15} \mathrm{H}_{11} \mathrm{O}_{4}, 255.0657\right)$.

$2^{\prime}$-O-trans-cinnamoylmangiferin (5; 2-(2'-O-trans-cinnamoyl)-C- $\beta$-D-glucopyranosyl-1,3,6,7-tetrahydroxyxanthone). orange powder $(\mathrm{MeOH}) ; \mathrm{m} . \mathrm{p}$. decomposes at 269.0-279.0 UV $(\mathrm{MeOH}) \lambda_{\max } 222,257,275$ (sh), 320 (sh), $366 \mathrm{~nm}$; IR $v_{\max }$ 3252, 1693, 1614, 1471, 1417, 1183, 1150, 1079, 815, 765, $706 \mathrm{~cm}^{-1}$; ${ }^{1} \mathrm{H}$ NMR (DMSO-d6, $\left.400 \mathrm{MHz}\right): \delta 13.87$ (s, 1H, 1-OH), 7.51 (m, 1H, H-3"), 7.51 (m, 1H, H-5 $5^{\prime \prime}$ ), 7.33 (s, 1H, H-8), 7.23 (d, $\left.16.0 \mathrm{~Hz}, 1 \mathrm{H}, \mathrm{H}-7^{\prime \prime}\right), 6.80$ (s, 1H, H-5), 6.36 (m, 1H, H-2" ), 6.36 (m, $\left.1 \mathrm{H}, \mathrm{H}-6^{\prime \prime}\right), 6.32$ (s, 1H, H-4), 6.31 (d, $\left.16.0 \mathrm{~Hz}, 1 \mathrm{H}, \mathrm{H}-8^{\prime \prime}\right)$, $5.55\left(\mathrm{~m}, 1 \mathrm{H}, \mathrm{H}-2^{\prime}\right), 5.04\left(\mathrm{~d}, 12.0 \mathrm{~Hz}, 1 \mathrm{H}, \mathrm{H}-1^{\prime}\right), 3.78$ (dd, 12.0, $\left.2.5 \mathrm{~Hz}, 1 \mathrm{H}, \mathrm{H}-6^{\prime}{ }_{\mathrm{B}}\right), 3.66\left(\mathrm{dd}, 12.0,4.0 \mathrm{~Hz}, 1 \mathrm{H}, \mathrm{H}^{\prime} 6^{\prime}{ }_{\mathrm{A}}\right), 3.64(\mathrm{~m}$, $\left.1 \mathrm{H}, \mathrm{H}-3^{\prime}\right), 3.51\left(\mathrm{~m}, 1 \mathrm{H}, \mathrm{H}-4^{\prime}\right), 3.44\left(\mathrm{~m}, 1 \mathrm{H}, \mathrm{H}-5^{\prime}\right) .{ }^{\mathrm{A}} \mathrm{C} \mathrm{NMR}$ (DMSO-d6, $100 \mathrm{MHz}$ ): $\delta 179.3$ (C, C-9), 165.0 (C, C-9" ${ }^{\prime \prime}$ ), 163.4 (C, C-3), 161.0 (C, C-1), 156.8 (C, C-4a), 153.9 (C, C-6), 151.1 (C, C-10a), 143.9 (C, C-7" $), 143.4$ (C, C-7), 133.9 (C, $\left.\mathrm{C}-1^{\prime \prime}\right), 129.9\left(\mathrm{C}, \mathrm{C}-4^{\prime \prime}\right), 128.5\left(\mathrm{C}, \mathrm{C}-2^{\prime \prime}\right), 128.5\left(\mathrm{C}, \mathrm{C}-6^{\prime \prime}\right), 127.7$ $\left(\mathrm{C}, \mathrm{C}-3^{\prime \prime}\right), 127.7\left(\mathrm{C}, \mathrm{C}-5^{\prime \prime}\right), 117.6\left(\mathrm{C}, \mathrm{C}-8^{\prime \prime}\right), 111.9$ (C, C-8a), 107.8 (C, C-8), 104.9 (C, C-2), 102.3 (C, C-5), 101.3 (C, C-9a), 94.0 (C, C-4), $80.9\left(\mathrm{C}, \mathrm{C}-5^{\prime}\right), 75.9\left(\mathrm{C}, \mathrm{C}-3^{\prime}\right), 72.4\left(\mathrm{C}, \mathrm{C}-2^{\prime}\right)$, $71.1\left(\mathrm{C}, \mathrm{C}-1^{\prime}\right), 70.2\left(\mathrm{C}, \mathrm{C}-4^{\prime}\right), 60.9\left(\mathrm{C}, \mathrm{C}-6^{\prime}\right)$; HRESI-MS m/z $551.1152[\mathrm{M}-\mathrm{H}]^{-}\left(\right.$calcd for $\left.\mathrm{C}_{28} \mathrm{H}_{23} \mathrm{O}_{12}, 551.1090\right)$.

\section{Results}

3.1. HPLC Analyses, Isolation, and Identification of Compounds from Fridericia formosa. HPLC-DAD analyses allowed identifying xanthones as major constituents in all the extracts, as inferred from their UV spectra that were registered online (Figure 1). UV spectra of 1,3,6,7-tetraoxygenated xanthones are characterized by the presence of three or more absorption bands of decreasing intensity [18]. A compound with retention time (RT) of 7.8 min was detected in all the ethanol extracts as the major constituent in extracts of stems and fruits.

Bioguided fractionation of $F$. formosa ethanol extracts led to the isolation of five compounds (Figure 2) which were identified by comparison with literature spectroscopic data $\left({ }^{1} \mathrm{H}\right.$ and ${ }^{13} \mathrm{C}$ NMR, DEPT-135 experiment, COSY, HMQC, $\mathrm{HMBC}$, IR, and MS). From the EtOH extract of leaves three compounds (1-3) were obtained. Compound 1 was identified as mangiferin, a C-glucosylxanthone $\left(\lambda_{\max } 239,258\right.$, 316 , and $365 \mathrm{~nm}$, HPLC-DAD online). Its identification was confirmed by HRMS and comparison of ${ }^{1} \mathrm{H}$ and ${ }^{13} \mathrm{C} N M R$ spectra with literature data [19]. Additionally, mangiferin (1) was also isolated from stems (EEAFS) and fruit (EEAFF) extracts. Compounds $\mathbf{2}$ and $\mathbf{3}$ were purified from leaves and fruits by Sephadex LH-20 gel filtration and preparative HPLC. The presence of the mangiferin chromophore for both compounds was indicated by their UV spectra. The protonated molecular ions $[\mathrm{M}+\mathrm{H}]^{+}$for 2 and $\mathbf{3}(\mathrm{m} / z 585.1084$ and $m / z 569.1144$, resp.) were determined by accurate positive HRESI-MS. A comparative analysis of fragment ions detected in experiments by HPLC/ESI-MS $\backslash$ MS showed that the only noticeable differences were associated with the cinnamic acid moieties attached to the mangiferin unity, caffeic acid in 2, and p-coumaric acid in 3 . The isolation of mangiferin cinnamic esters from Fridericia samydoides and F. patellifera was previously reported $[20,21]$ and a comparative analysis of these NMR data confirmed the identification of compounds 2 and 3 as $2^{\prime}$-O-trans-caffeoylmangiferin and $2^{\prime}-O$ trans-coumaroylmangiferin, respectively. The position of the cinnamic ester group in the glucose unity was proved by longrange $\mathrm{HMBC}$ correlation between the ester carbonyl group and the glucosyl hydrogen: $\delta_{\mathrm{C}} 165.2$ and $\mathrm{H}-2^{\prime} \delta_{\mathrm{H}} 5.65$, in compound 2 , and $\delta_{\mathrm{C}} 165.8$ and $\mathrm{H}-2^{\prime} \delta_{\mathrm{H}} 5.56$, in compound 3.

Additional quantity of mangiferin (1) was obtained as an unsoluble fraction when methanol was added to the dried stem ethanol extract. Fractionation of the filtrate (AFSMW2) by preparative RP-HPLC afforded compound 4 whose spectral data (UV, IR, ${ }^{1} \mathrm{H}$ and ${ }^{13} \mathrm{C}$ NMR) and comparison with literature data [22] allowed its identification as the flavonoid chrysin (4).

Finally, column chromatographic fractionation of the ethanol fruit extract afforded four C-glucosylxanthones which were shown to be identical to those obtained from the leaves extract, mangiferin (1), caffeoyl mangiferin (2), and coumaroyl mangiferin (3), besides compound 5, and MM $542 \mathrm{Da}$, which might correspond to a mangiferin cinnamic ester, was confirmed by NMR data. Two regioisomers, $2^{\prime}$-O-trans-cinnamoylmangiferin and $3^{\prime}$-O-transcinnamoylmangiferin, have been previously isolated from stems and leaves extracts of $F$. samydoides and F. patellifera, respectively $[20,21]$. A comparison of ${ }^{13} \mathrm{C}$ and ${ }^{1} \mathrm{H}$ NMR data, including two-dimensional COSY, HSQC, and $\mathrm{HMBC}$, with those previously reported [20] allowed the identification of compound 5 as $2^{\prime}$-O-trans-cinnamoylmangiferin.

3.2. Bioguided Fractionation of Leaves, Stems, and Fruits Ethanol Extracts from Fridericia formosa. Confirming previously published results [8], the ethanol extracts from leaves (EEFFL), stems (EEFFS), and fruits (EEFFF) of F. formosa showed antiviral activity against EMCV, HSV-1, and VACVWR with $\mathrm{EC}_{50}$ values in the range of $85.6 \pm 4.1$ to 147.8 $\pm 2.4 \mu \mathrm{g} / \mathrm{mL}$ (Table 1 and Figure 3). Furthermore, these extracts were evaluated against DENV-2 and disclosed good antidengue activity with $\mathrm{EC}_{50}$ values ranging from $13.1 \pm 1.6$ to $42.6 \pm 2.3 \mu \mathrm{g} / \mathrm{mL}$ (Table 1 ).

An aliquot of the leaves extract (EEFFL, 10.0 g) was submitted to bioguided fractionation. Initially, addition of cold methanol to EEFFL led to mangiferin (1), as an 


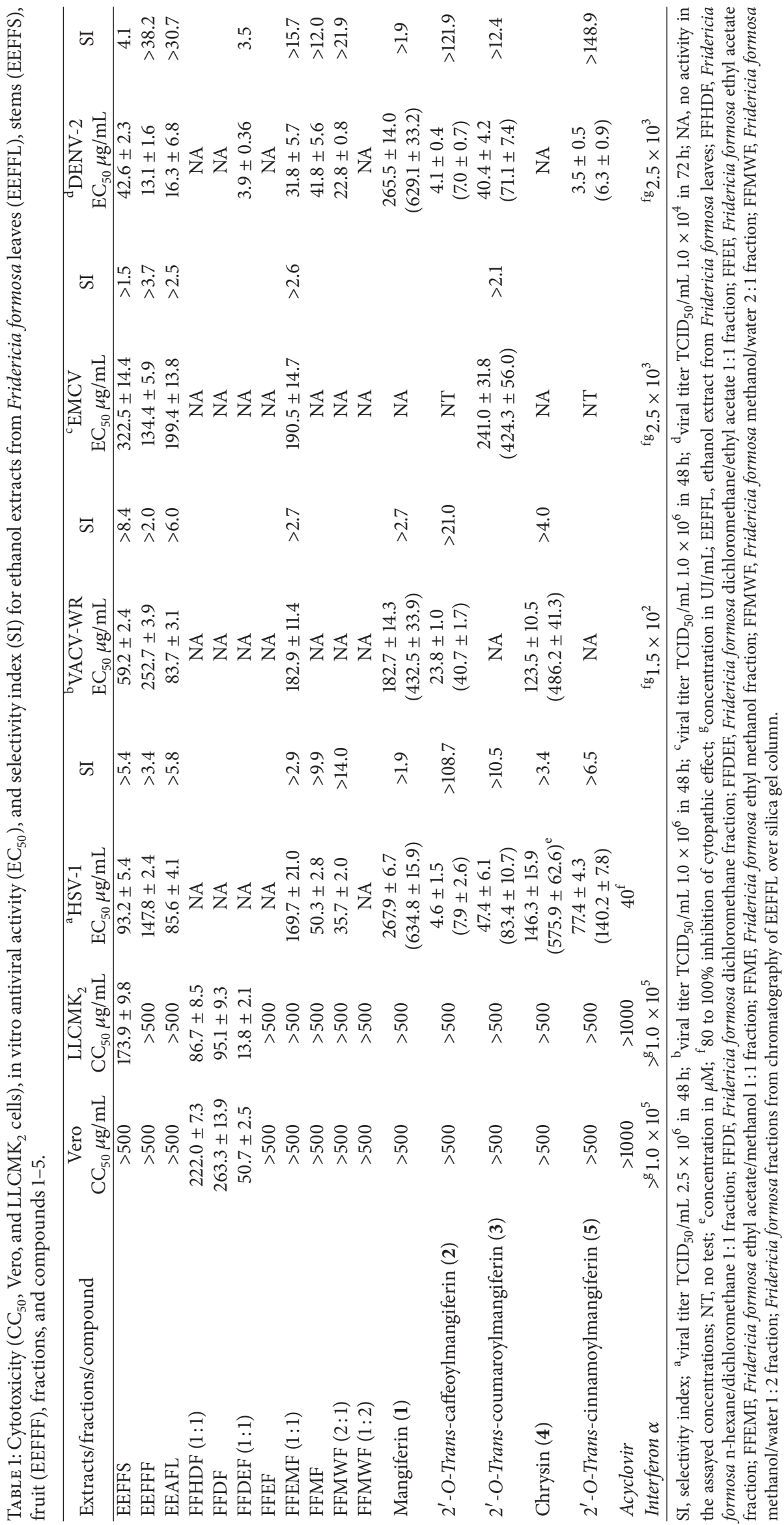




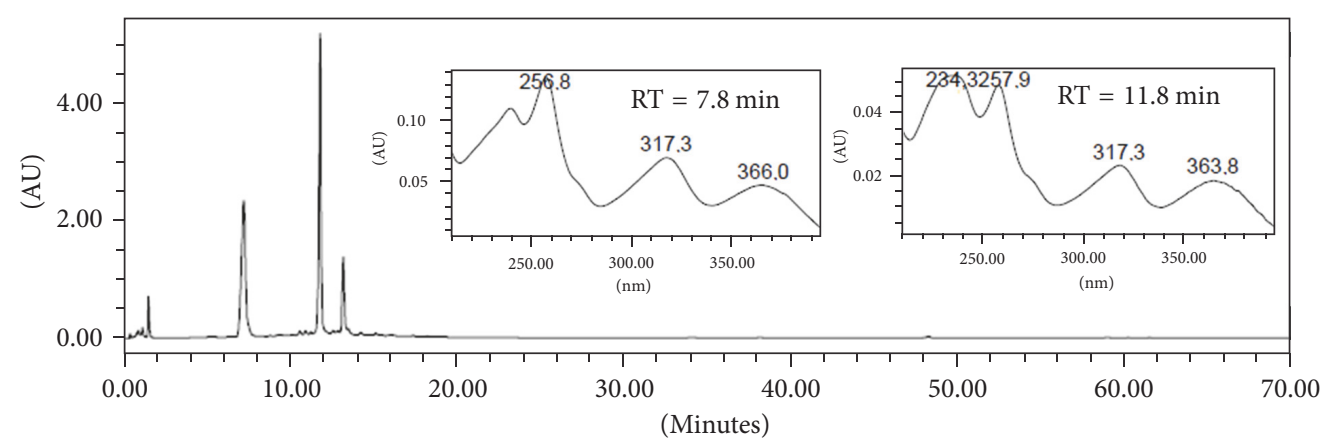

(a)

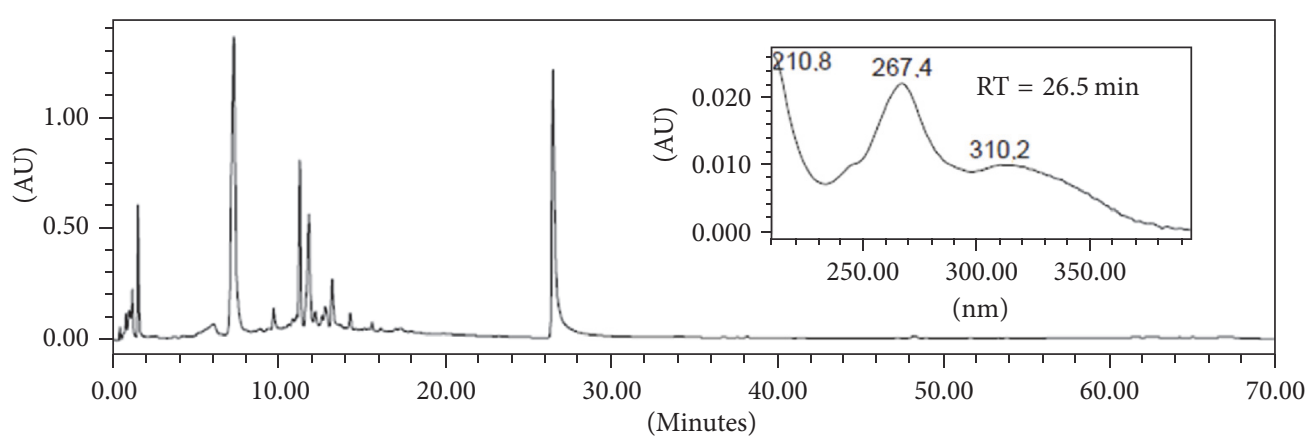

(b)

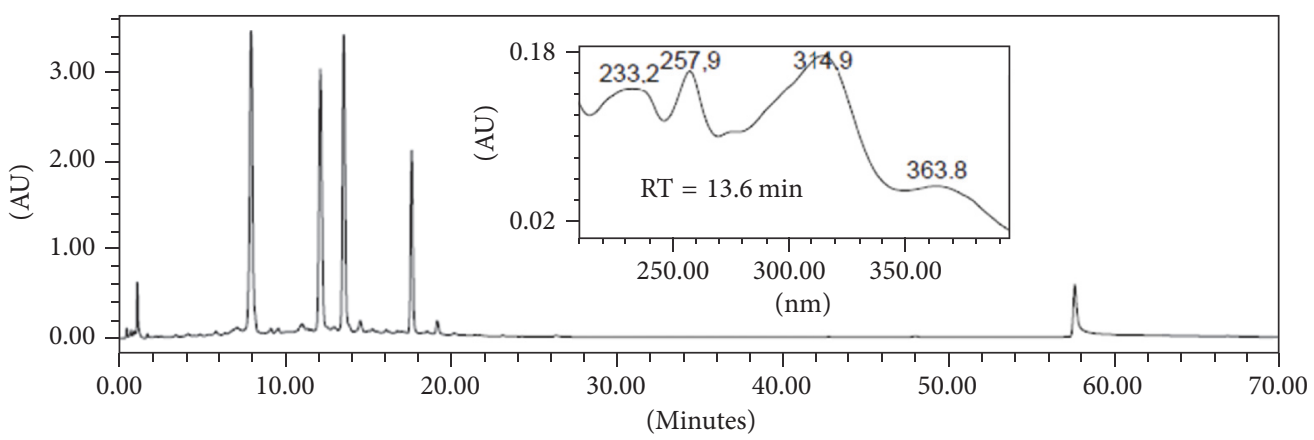

(c)

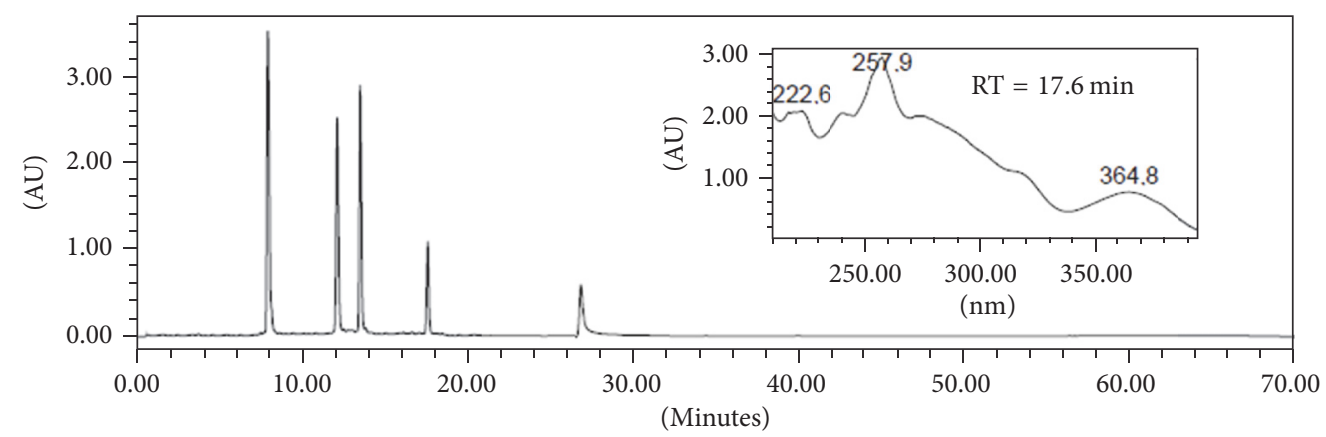

(d)

FIGURE 1: RP-HPLC-DAD fingerprints for the crude ethanol extracts from (a) ethanol extract from Fridericia formosa leaves (EEFFL) with mangiferin $(\mathrm{RT}=7.8 \mathrm{~min})$ and $2^{\prime}$-O-trans-caffeoylmangiferin $(\mathrm{RT}=11.8 \mathrm{~min}) \mathrm{UV}$ spectrum registered online detection $350 \mathrm{~nm}$, (b) ethanol extract from Fridericia formosa stems (EEFFS) with UV spectra registered online for peak corresponding to chrysin (RT $=$ $26.8 \mathrm{~min}$ ), (c) ethanol extract from Fridericia formosa fruits (EEFFF) with UV spectra registered online for peak corresponding to $2^{\prime}$ - $O$ trans-coumaroylmangiferin ( $\mathrm{RT}=13.6 \mathrm{~min}$ ), and $(\mathrm{d})$ for a mixture of the isolated compounds with UV spectra registered online for peak corresponding to $2^{\prime}$-O-trans-cinnamoylmangiferin $(\mathrm{RT}=17.6 \mathrm{~min})$. Detection: $350 \mathrm{~nm}$. Chromatographic conditions: see Experimental. 


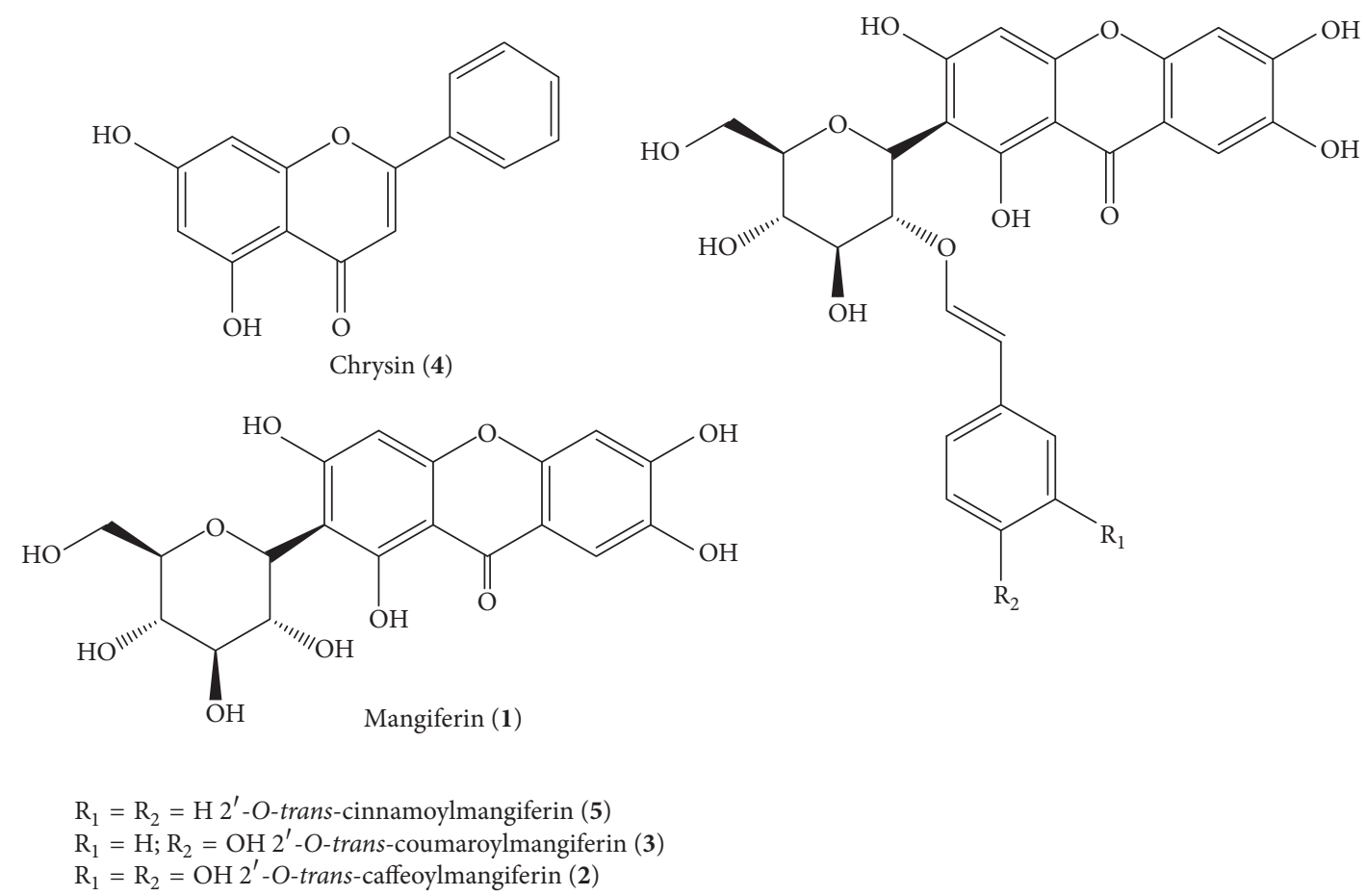

Figure 2: Chemical structures of mangiferin (1), 2'-O-trans-caffeoylmangiferin (2), $2^{\prime}$-O-trans-coumaroylmangiferin (3), chrysin (4), and $2^{\prime}$-O-trans-cinnamoylmangiferin (5).

unsoluble fraction $(1.9 \mathrm{~g})$, and to a methanol soluble fraction (FFLMW). Mangiferin (1) was tested against all the four virus samples and showed a low antiviral effect (Table 1). FFLMW was subjected to a chromatographic fractionation through a silica gel column employing as eluents n-hexane/ $\mathrm{CH}_{2} \mathrm{Cl}_{2}(1: 1), \mathrm{CH}_{2} \mathrm{Cl}_{2}, \mathrm{CH}_{2} \mathrm{Cl}_{2} /$ EtOAc $(1: 1)$, EtOAc/MeOH $(2: 1)$, EtOAc/MeOH $(1: 2), \mathrm{MeOH}$, $\mathrm{MeOH} / \mathrm{H}_{2} \mathrm{O}(2: 1)$, and $\mathrm{MeOH} / \mathrm{H}_{2} \mathrm{O}(1: 2)$ fractions that were assayed against DENV-2, EMCV, HSV-1, and VACV-WR. Three fractions were active against HSV-1. The EtOAc/MeOH $(2: 1)$ fraction was the only one active against VACV-WR and EMCV. Best results were observed against DENV-2 for four active fractions with $\mathrm{EC}_{50}$ ranging from $3.9 \pm 0.4$ to 41.8 $\pm 5.6 \mu \mathrm{g} / \mathrm{mL}$ (Table 1). Fractionation of the EtOAc/MeOH $(2: 1)$ fraction through a Sephadex LH20 column and preparative RP-HPLC afforded two xanthones: $2^{\prime}$-O-transcaffeoylmangiferin (2) and $2^{\prime}$-O-trans-coumaroylmangiferin (3). Xanthone 2 showed high activity against DENV-2, HSV-1, and VACV-WR $\left(\mathrm{EC}_{50}\right.$ of $4.1 \pm 0.4 \mu \mathrm{g} / \mathrm{mL}, 4.6 \pm$ $1.5 \mu \mathrm{g} / \mathrm{mL}$, and $23.8 \pm 1.0 \mu \mathrm{g} / \mathrm{mL}$, resp.) while $2^{\prime}$-O-transcoumaroylmangiferin (3) was active against DENV-2, EMCV, and HSV-1 but with lower $\mathrm{EC}_{50}$ values (Table 1 and Figure 4).

EEFFS (10.0 g) was also submitted to bioguided fractionation. Initially, addition of cold methanol to the crude ethanol extract led to the separation of mangiferin (1) (405.0 mg), as the unsoluble fraction. Fractionation of the methanol soluble fraction (FFCMW) by preparative RP-HPLC afforded chrysin (4) that showed low activity against HSV-1 and VACV-WR (Table 1 and Figure 4).
Finally, bioguided fractionation of EEFFS (10.0 g) by extraction with cold methanol led to mangiferin (1) $(80.7 \mathrm{mg}$ ), as an unsoluble part, and FFFMW (methanol soluble fraction) that, on fractionation by preparative RPHPLC, afforded mangiferin (1), 2'-O-trans-caffeoylmangiferinn (2), $2^{\prime}$-O-trans-coumaroylmangiferin (3), and $2^{\prime}-O$ trans-cinnamoylmangiferin (5). The last compound (5) was highly active against DENV-2 virus $\left(\mathrm{EC}_{50} 3.5 \pm 0.5 \mu \mathrm{g} / \mathrm{mL}\right)$ and moderately active against HSV-1 (EC E0 $\left._{50} 77.4 \pm 4.3\right)$ (Table 1 and Figure 4).

\section{Discussion}

Phytochemical investigation of EtOH extracts of leaves, stems, and fruits from $F$. formosa led to the isolation of five compounds. Four of them were identified as Cglucosylxanthones, namely, mangiferin (1), along with three cinnamoyl esters of mangiferin $(2,3$, and 5), and one flavonoid, chrysin (4) (Figure 2). Chrysin (4) was isolated from the stems extract and showed low antiviral activity against VACV-WR and HSV-1 with $\mathrm{EC}_{50}>100 \mu \mathrm{g} / \mathrm{ml}$ and did not inhibit the replication cycle of DENV-2 and EMCV. Good activity of this flavonoid against HSV-1 with an $\mathrm{EC}_{50} 2.5 \mu \mathrm{M}$ was previously reported [23] and our negative result might be related to difference in susceptibility of strains. This flavonoid is present in other species of the Bignoniaceae family, such as F. samydoides [24]. Previously isolated from Oroxylum indicum (Bignoniaceae), it is reported as disclosing in vitro anti-inflammatory and anticancer effects [25-27]. Marketed as a bodybuilding supplement it is claimed to increase 


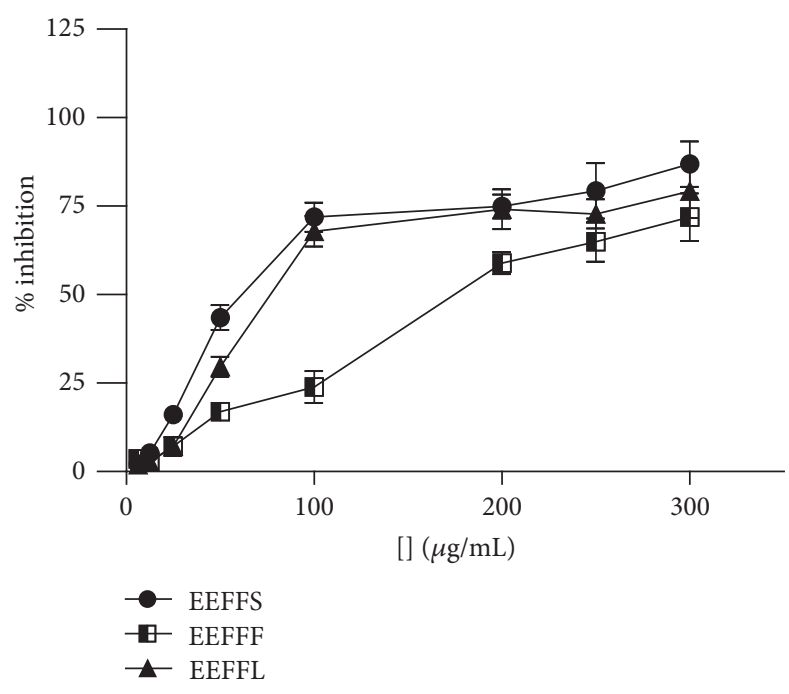

(a)

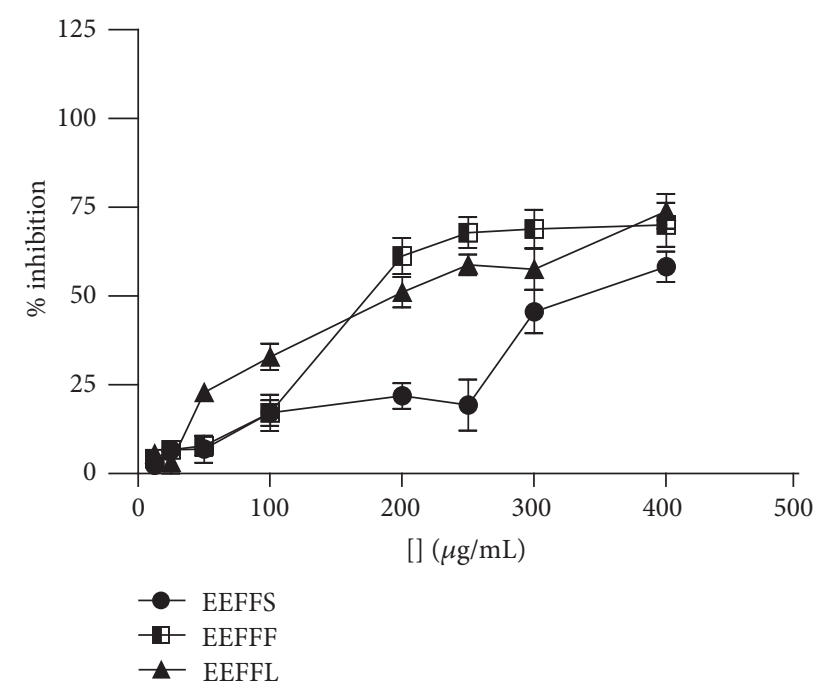

(c)

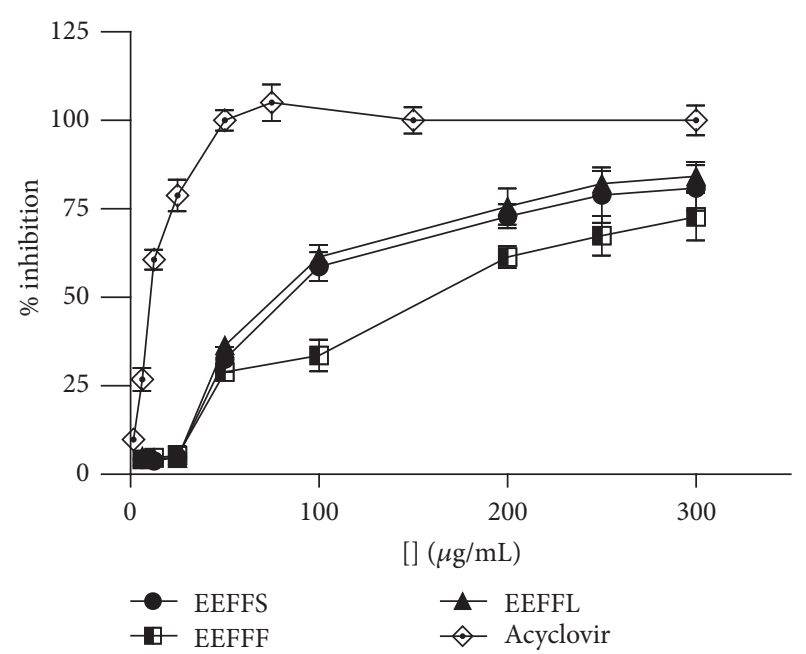

(b)

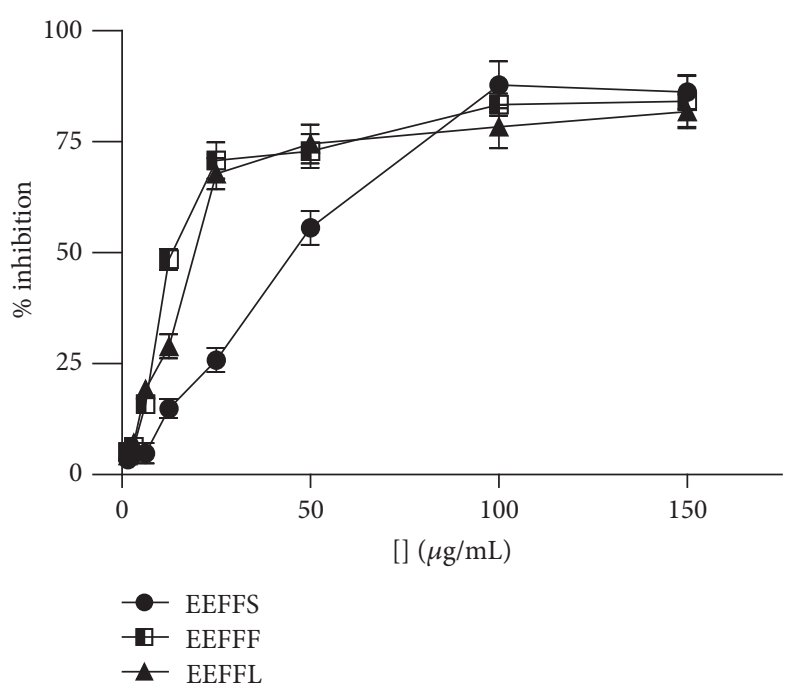

(d)

FIGURE 3: Dose-response curves for antiviral activity of ethanol extracts from Fridericia formosa (a) leaves (EEFFL), stems (EEFFS), and fruits (EEFFF) against VAC-WR; (b) EEFFL, EEFFS, and EEFFF against HSV-1; (c) EEFFL, EEFFS, and EEFFF against EMCV; (d) EEFFL, EEAFS, and EEFFF against DENV-2.

testosterone levels or stimulate testosterone production; however, clinical tests have shown no effect on testosterone levels in men [28].

As shown in Figure 2, the four xanthones isolated from the extracts of leaves, stems, and fruits of $A$. formosa were identified as mangiferin (1), $2^{\prime}$-O-trans-caffeoylmangiferin (2), $2^{\prime}$-O-trans-coumaroylmangiferin (3), and $2^{\prime}$-O-transcinnamoylmangiferin (5). The antiviral effect of these compounds was evaluated against DENV-2, EMCV, HSV-1, and VACV-WR (Figure 3). Excepting mangiferin (1), the other three xanthones showed good antiviral effects and inhibited the replication cycle of DENV-2, HSV-1, and VACV-WR (Table 1 and Figure 4).

Mangiferin (1) is mainly obtained from mango tree (Mangifera indica) and preclinical studies showed that it exhibits antidiabetic, antioxidant, antiviral, cardiotonic, hypotensive, and anti-inflammatory properties [29]. The biological activities of mangiferin have been attributed to modulating expression of a large number of genes that are critical for the regulation of apoptosis, viral replication, inflammation, and various autoimmune diseases [30]. Furthermore mangiferin disclosed low cytotoxicity and good inhibitory activity on HIV-1 replication in a dose dependent manner [30, 31]. Mechanism studies revealed that mangiferin might inhibit the HIV-1 protease and is, therefore, a novel nonpeptide protease inhibitor of HIV protease $[30,31]$.

Recent data on biological activity of mangiferin cinnamoyl esters showed that they are antioxidant agents similar to mangiferin and are antiplasmodial with moderate activity in vitro against Plasmodium falciparum 3D7 clone, which is chloroquine-sensitive $\left(\mathrm{IC}_{50} 18.1\right.$ to $\left.26.5 \mu \mathrm{M}\right)$ [21]. 


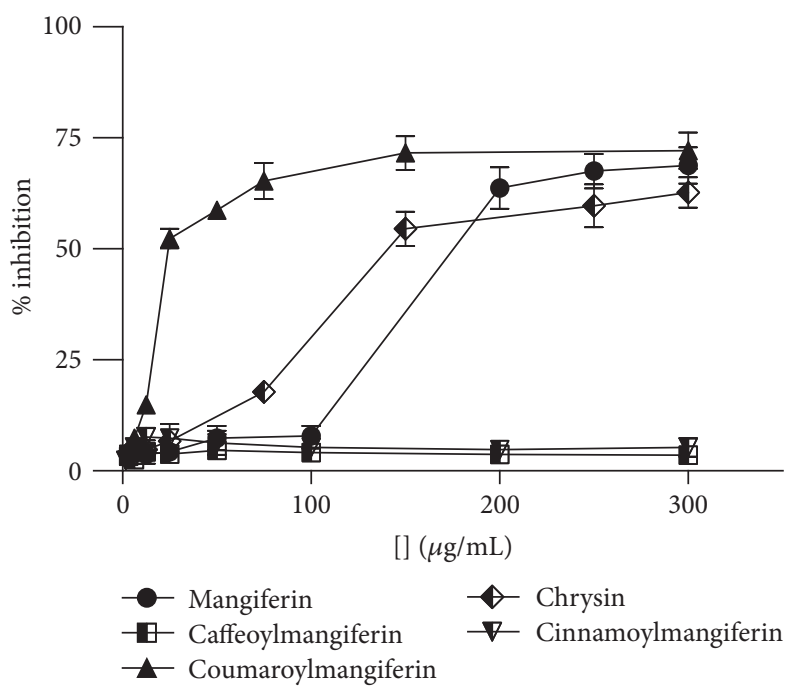

(a)

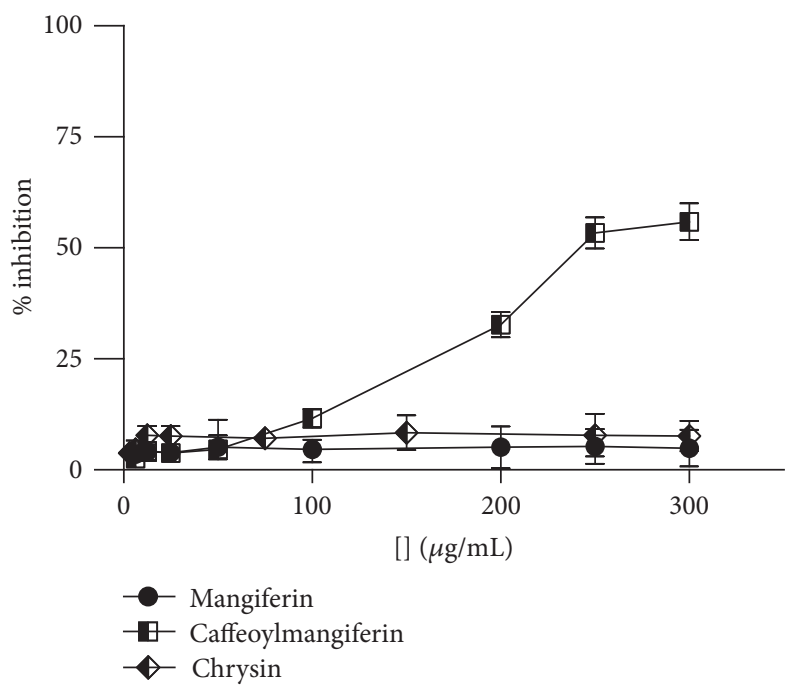

(c)

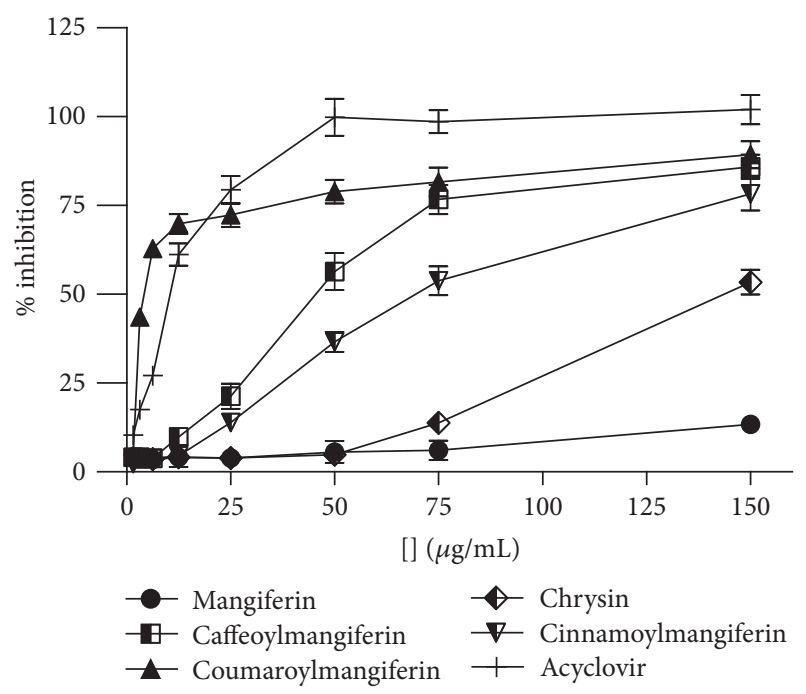

(b)

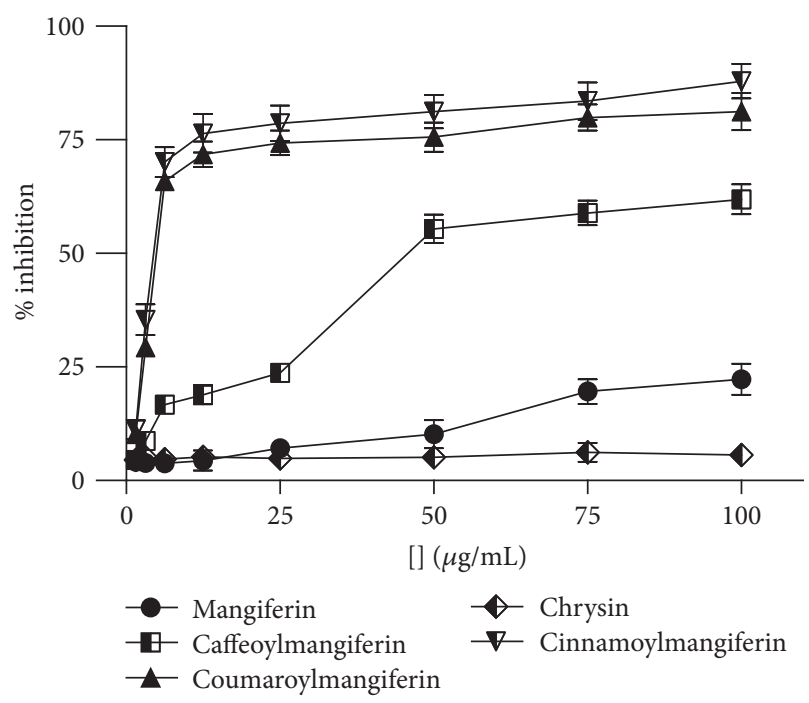

(d)

FIGURE 4: Dose-response curves for antiviral activity of mangiferin (1), $2^{\prime}$-O-trans-caffeoylmangiferin (2), $2^{\prime}$-O-trans-coumaroylmangiferin (3), chrysin (4), and 2'-O-trans-cinnamoylmangiferin (5). (a) Against VAC-WR; (b) against HSV-1; (c) against EMCV; (d) against DENV-2.

Recently, a total of twenty xanthones were isolated from Swertia mussotii (Gentianaceae) and their antiviral activity was evaluated [32]. Eight of these xanthones exhibited significant activity against hepatitis B virus inhibiting DNA replication with $\mathrm{EC}_{50}$ values from $0.01 \mathrm{mM}$ to $0.13 \mathrm{mM}$ [32]. Additionally, the xanthones norbellidifolin, 1,5,8-trihydroxy-3-methoxyxanthone, and 2-C- $\beta$ - $D$ glucopyranosyl-1,3,7-trihydroxyxanthone showed remarkable activity with $\mathrm{EC}_{50}$ values of $0.77,>0.98$, and $0.21 \mathrm{mM}$ for hepatitis B surface antigen ( $\mathrm{HBsAg}$ ) and $<0.62,0.35$, and $0.04 \mathrm{mM}$ for hepatitis B antigen (HBeAg), respectively [32]. Besides, euxanthone, from Garcinia oblongifolia (Clusiaceae) leaves extract, disclosed significant activity in vitro against Enterovirus 71, with $\mathrm{EC}_{50}$ value of $12.2 \mu \mathrm{M}$ [33]. In addition, the selectivity index of this compound was 3.0 in relation to the cytotoxicity to Vero cells $\left(\mathrm{CC}_{50} 36.6 \mu \mathrm{M}\right)$ [33]. Molecular docking studies of 272 xanthones for interactions with a group of seven fungal and two viral enzymes showed that prenylated xanthones are important hits for inhibition of the selected enzymes [34]. In general, prenylated xanthones were able to establish significantly stronger complexes with the tested enzymes [34]. Some compounds were pointed out as potential inhibitors for those enzymes, including nigrolineaxanthone and latisxanthone $\mathrm{D}$ as probably potent inhibitors of HIV-1 reverse transcriptase [34]. Xanthones have important advantages as potential antiviral agents because of their availability as natural compounds and the possibility of being easily synthesized and also for the demonstrated interaction with some important microorganisms targets [34]. 


\section{Conclusions}

Our results reveal that $F$. formosa is a rich source of mangiferin (1) ( $\cong 47 \mathrm{~g} \cdot \mathrm{kg}^{-1}$ of dry leaves), a C-glucosyl xanthone with several therapeutic and cosmetic uses [30]. Its content in F. formosa is higher than in mango tree (Mangifera indica), its usual source ( $\cong 1.7 \mathrm{~g} \cdot \mathrm{kg}^{-1}$ of dry peel) [35]. However, mangiferin was practically inactive against the virus assayed. On the other hand, minor constituents, represented by mangiferin cinnamoyl esters, seem to be the main responsible constituents for the antiviral activity previously reported for extracts of different botanical parts of this species [8]. Special attention is called for the IS of these xanthone derivatives, particularly for the caffeoyl (2) and cinnamoyl (5) esters, with IS $>100$. Our findings are the first report on the chemical and antiviral activity of $F$. formosa constituents. Our results are in line with the traditional use of Fridericia species as antiinfectious agents in different South American countries [1,9] and might be of interest for the development of standardized antiviral phytomedicines.

\section{Conflicts of Interest}

The authors declare no conflicts of interest.

\section{Acknowledgments}

Thanks are due to Dr. J. A. Lombardi, Departamento de Botânica, Instituto de Biociências, UNESP, Rio Claro, Brazil, for collection and taxonomical determination of F. formosa. This work was supported by funds from FAPEMIG (Fundação de Amparo à Pesquisa do Estado de Minas Gerais, Brazil), Process no. CDS-APQ-00270-13, and CAPES (Coordenação de Aperfeiçoamrnto de Pessoal de Ensino Superior, Brazil) and CNPq (Conselho Nacional de Desenvolvimento Científico e Tecnológico, Brazil) are also acknowledged for fellowships to Geraldo Célio Brandão, Erna G. Kroon, and Alaíde Braga Oliveira.

\section{References}

[1] G. C. Brandão, E. G. Kroon, D. E. R. Souza, J. D. S. Filho, and A. B. Oliveira, "Chemistry and antiviral activity of Arrabidaea pulchra (Bignoniaceae)," Molecules, vol. 18, no. 8, pp. 9919-9932, 2013.

[2] World Health Organization, Dengue and Severe Dengue, 2016, http://www.who.int/mediacentre/factsheets/fs117/en/.

[3] R. V. Gibbons, “Dengue conundrums," International Journal of Antimicrobial Agents, vol. 36, no. 1, pp. S36-S39, 2010.

[4] World Health Organization, "Herpes simplex virus," http:// www.who.int/mediacentre/factsheets/fs400/en/.

[5] M. S. Oberste, E. Gotuzzo, P. Blair et al., "Human febrile illness caused by encephalomyocarditis virus infection, peru," Emerging Infectious Diseases, vol. 15, no. 4, pp. 640-646, 2009.

[6] J. C. Quixabeira-Santos, M. L. G. Medaglia, C. A. Pescador, and C. R. Damaso, "Animal movement and establishment of vaccinia virus Cantagalo Strain in Amazon Biome, Brazil," Emerging Infectious Diseases, vol. 17, no. 4, pp. 726-729, 2011.
[7] G. S. Trindade, M. I. C. Guedes, B. P. Drumond et al., "Zoonotic vaccinia virus: Clinical and immunological characteristics in a naturally infected patient," Clinical Infectious Diseases, vol. 48, no. 3, pp. e37-e40, 2009.

[8] G. C. Brandão, E. G. Kroon, J. R. Dos Santos, J. R. Stehmann, J. A. Lombardi, and A. Braga De Oliveira, "Antiviral activity of Bignoniaceae species occurring in the state of Minas Gerais (Brazil): Part 1," Letters in Applied Microbiology, vol. 51, no. 4, pp. 469-476, 2010.

[9] G. C. Brandão, E. G. Kroon, J. R. dos Santos, J. R. Stehmann, J. A. Lombardi, and A. B. de Oliveira, "Antiviral activities of plants occurring in the state of Minas Gerais, Brazil. Part 2. Screening bignoniaceae species," Brazilian Journal of Pharmacognosy, vol. 20, no. 5, pp. 742-750, 2010.

[10] G. C. Brandão, E. G. Kroon, J. R. dos Santos, J. R. Stehmann, J. A. Lombardi, and A. B. Oliveira, "Antiviral activity of plants occurring in the state of minas gerais (Brazil): Part III," Journal of Chemical and Pharmaceutical Research, vol. 3, no. 4, pp. 223236, 2011.

[11] L. R. Simões, G. M. Maciel, G. C. Brandão, E. G. Kroon, R. O. Castilho, and A. B. Oliveira, "Antiviral activity of Distictella elongata (Vahl) Urb. (Bignoniaceae), a potentially useful source of anti-dengue drugs from the state of Minas Gerais, Brazil," Letters in Applied Microbiology, vol. 53, no. 6, pp. 602-607, 2011.

[12] G. C. Brandão, E. G. Kroon, A. W. Matosa, J. D. Souza Filho, and A. B. Oliveira, "Bioguided isolation of an antiviral compound from Xylophragma myrianthum (Cham.) Sprague (Bignoniaceae Juss.)," Revista Fitos, vol. 8, no. 2, pp. 125-136, 2013.

[13] D. J. De Rodriguez, J. Chulia, C. M. O. Simoes, M. Amoros, A. M. Mariotte, and L. Girre, "Search for 'in vitro' antiviral activity of a new isoflavonec glycoside from ulex europaeus," Planta Medica, vol. 56, no. 1, pp. 59-62, 1990.

[14] P. R. Twentyman and M. Luscombe, "A study of some variables in a tetrazolium dye (MTT) based assay for cell growth and chemosensitivity," British Journal of Cancer, vol. 56, no. 3, pp. 279-285, 1987.

[15] L. A. Betancur-Galvis, S. J. H. Granados, A. Salazar, and J. E. Ossa, "Antitumor and antiviral activity of colombian medicinal plant extracts," Memorias do Instituto Oswaldo Cruz, vol. 94, no. 4, pp. 531-535, 1999.

[16] R. A. Finnegan, R. A. Stephani, G. Ganguli, S. N. Ganguly, and A. K. Bhattacharya, "Occurrence of mangiferin in Hiptage madablota geartn," Journal of Pharmaceutical Sciences, vol. 57, no. 6, pp. 1039-1040, 1968.

[17] M. P. Yuldashev and A. Karimov, "Flavonoids of Scutellaria immaculata roots," Chemistry of Natural Compounds, vol. 41, no. 1, pp. 32-34, 2005.

[18] R. K. Chaudhuri and S. Ghosal, "Xanthones of Canscora decussata schult," Phytochemistry, vol. 10, no. 10, pp. 2425-2432, 1971.

[19] S. Catalano, S. Luschi, G. Flamini, P. L. Cioni, E. M. Nieri, and I. Morelli, "A xanthone from Senecio mikanioides leaves," Phytochemistry, vol. 42, no. 6, pp. 1605-1607, 1996.

[20] P. Mendonça Pauletti, I. Castro-Gamboa, D. H. Siqueira Silva et al., "New Antioxidant C-Glucosylxanthones from the Stems of Arrabidaea samydoides," Journal of Natural Products, vol. 66, no. 10, pp. 1384-1387, 2003.

[21] F. Martin, A.-E. Hay, D. Cressend et al., "Antioxidant Cglucosylxanthones from the leaves of Arrabidaea patellifera," Journal of Natural Products, vol. 71, no. 11, pp. 1887-1890, 2008. 
[22] P. K. Agrawal, Carbon-13 NMR of Flavonoids, vol. 28, Elsevier, Amsterdam, Nederland, IST edition, 1989.

[23] S.-Y. Lyu, J.-Y. Rhim, and W.-B. Park, "Antiherpetic activities of flavonoids against herpes simplex virus type 1 (HSV-1) and type 2 (HSV-2) in vitro," Archives of Pharmacal Research, vol. 28, no. 11, pp. 1293-1301, 2005.

[24] P. M. Pauletti, V. s. Bolzani, and M. C. M. Young, "Chemical constituents of Arrabidaea samydoides (Bignoniaceae)," Quimica Nova, vol. 26, no. 5, pp. 641-643, 2003.

[25] L.-J. Chen, D. E. Games, and J. Jones, "Isolation and identification of four flavonoid constituents from the seeds of Oroxylum indicum by high-speed counter-current chromatography," Journal of Chromatography A, vol. 988, no. 1, pp. 95-105, 2003.

[26] K. J. Woo, Y.-J. Jeong, H. Inoue, J.-W. Park, and T. K. Kwon, "Chrysin suppresses lipopolysaccharide-induced cyclooxygenase-2 expression through the inhibition of nuclear factor for IL6 (NF-IL6) DNA-binding activity," FEBS Letters, vol. 579, no. 3 , pp. 705-711, 2005.

[27] K. J. Woo, Y.-J. Jeong, J.-W. Park, and T. K. Kwon, "Chrysininduced apoptosis is mediated through caspase activation and Akt inactivation in U937 leukemia cells," Biochemical and Biophysical Research Communications, vol. 325, no. 4, pp. 12151222, 2004.

[28] C. Gambelunghe, R. Rossi, M. Sommavilla et al., "Effects of chrysin on urinary testosterone levels in human males," Journal of Medicinal Food, vol. 6, no. 4, pp. 387-390, 2003.

[29] K. Shah, M. Patel, R. Patel, and P. Parmar, "Mangifera indica (mango)," Pharmacognosy Reviews, vol. 4, no. 7, pp. 42-48, 2010.

[30] M. Telang, S. Dhulap, A. Mandhare, and R. Hirwani, “Therapeutic and cosmetic applications of mangiferin: A patent review," Expert Opinion on Therapeutic Patents, vol. 23, no. 12, pp. 1561-1580, 2013.

[31] R.-R. Wang, Y.-D. Gao, C.-H. Ma et al., "Mangiferin, an antiHIV-1 agent targeting protease and effective against resistant strains," Molecules, vol. 16, no. 5, pp. 4264-4277, 2011.

[32] T.-W. Cao, C.-A. Geng, Y.-B. Ma et al., "Xanthones with antihepatitis B virus activity from Swertia mussotii," Planta Medica, vol. 79, no. 8, pp. 697-700, 2013.

[33] H. Zhang, L. Tao, and W.-W. Fu, "Prenylated benzoylphloroglucinols and xanthones from the leaves of Garcinia oblongifolia with antienteroviral activity," Journal of Natural Products, vol. 77, no. 4, pp. 1037-1046, 2014.

[34] F. A. Bernal and E. Coy-Barrera, "Molecular docking and multivariate analysis of xanthones as antimicrobial and antiviral agents," Molecules, vol. 20, no. 7, pp. 13165-13204, 2015.

[35] M. Masibo and H. Qian, "Major mango polyphenols and their potential significance to human health," Comprehensive Reviews in Food Science and Food Safety, vol. 7, no. 4, pp. 309-319, 2008. 


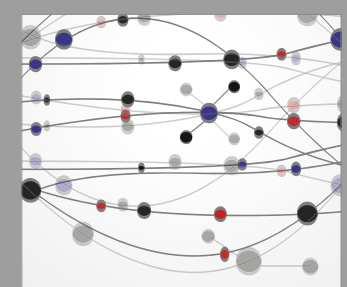

The Scientific World Journal
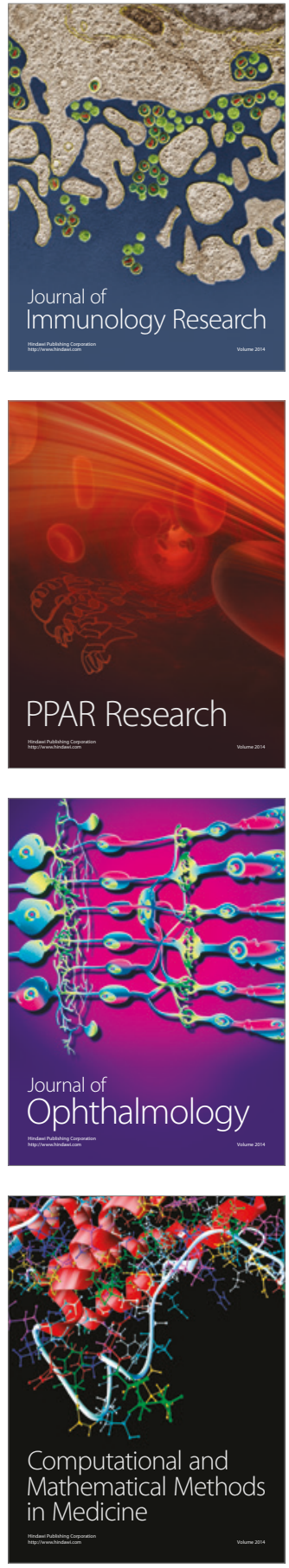

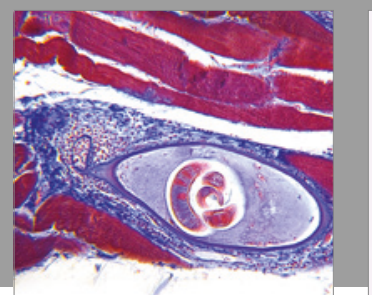

Gastroenterology Research and Practice
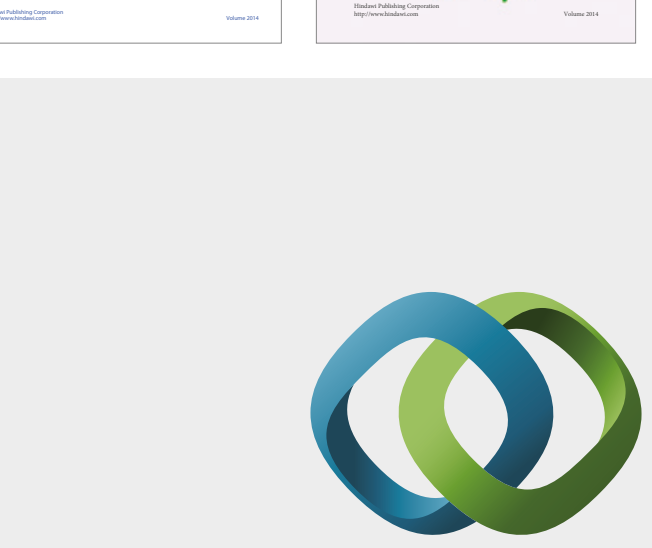

\section{Hindawi}

Submit your manuscripts at

https://www.hindawi.com
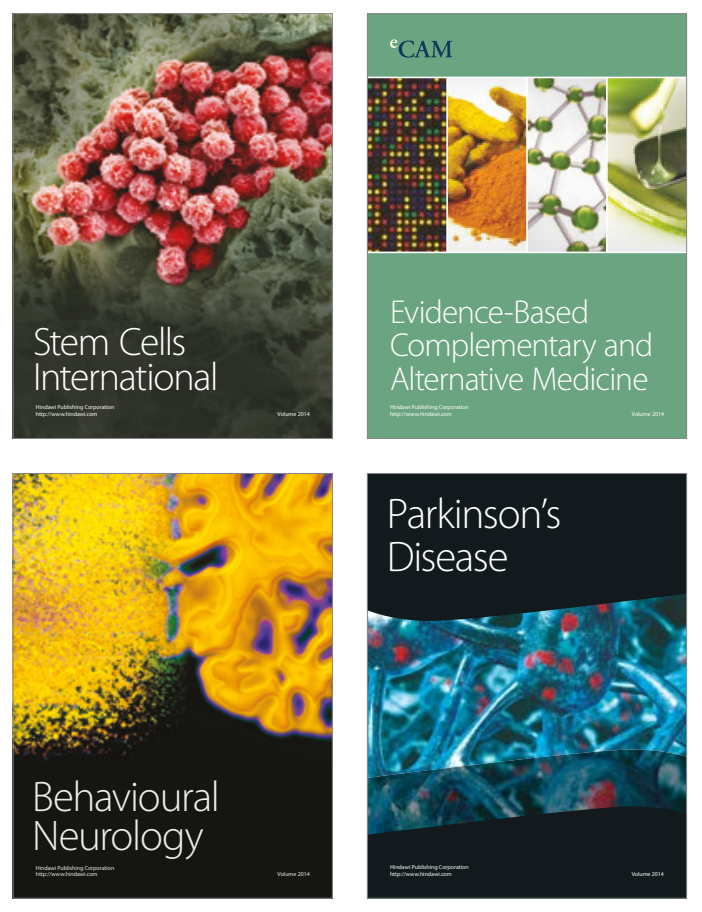
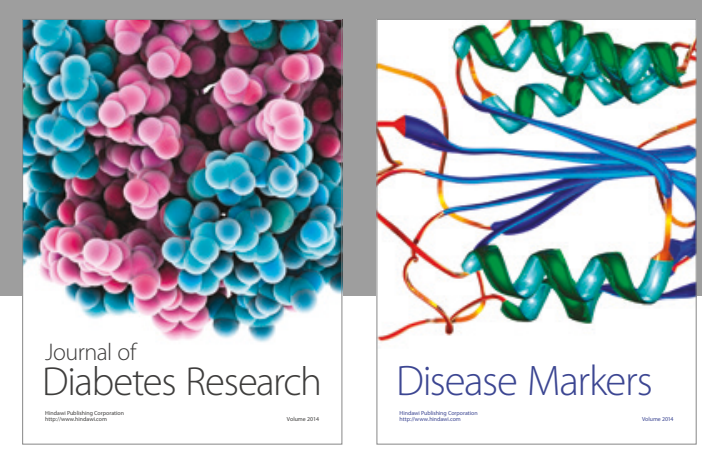

Disease Markers
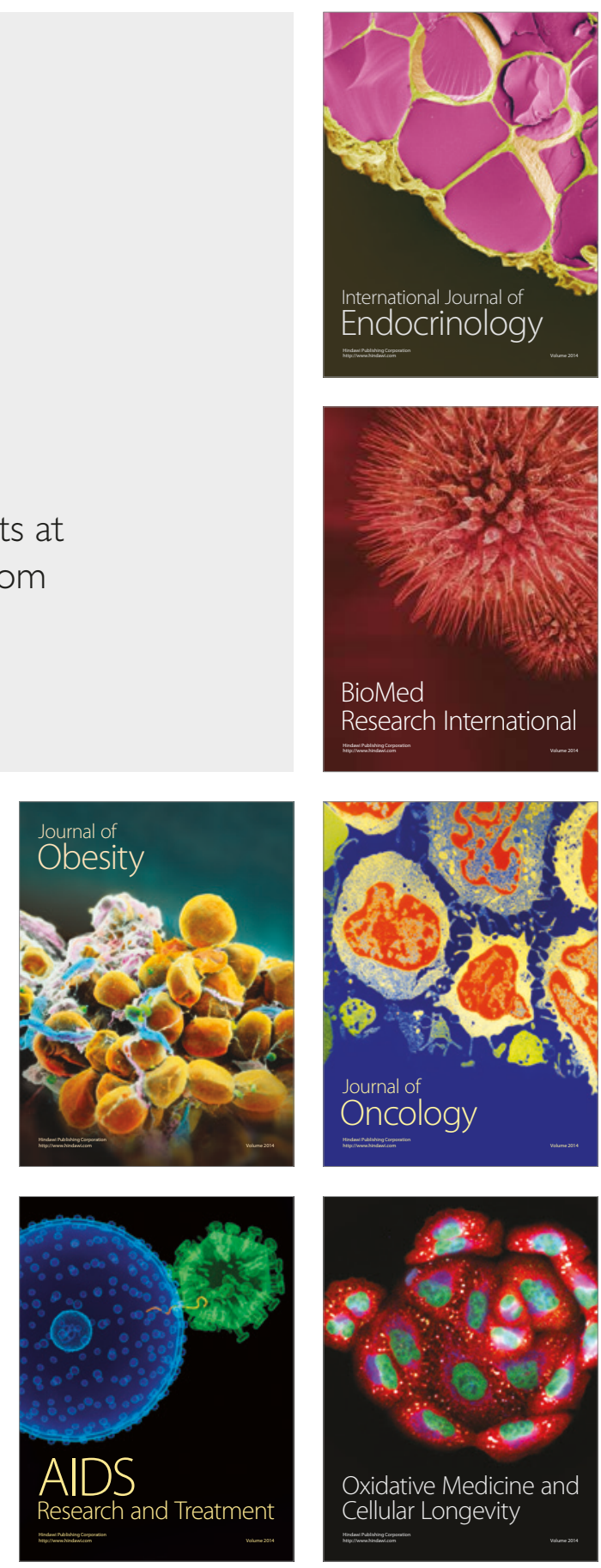